\title{
PERMUTATION STATISTICS RELATED TO A CLASS OF NONCOMMUTATIVE SYMMETRIC FUNCTIONS AND GENERALIZATIONS OF THE GENOCCHI NUMBERS
}

\author{
FLORENT HIVERT, JEAN-CHRISTOPHE NOVELLI, \\ LENNY TEVLIN, AND JEAN-YVES THIBON
}

\begin{abstract}
We prove conjectures of the third author [L. Tevlin, Proc. FPSAC'07, Tianjin] on two new bases of noncommutative symmetric functions: the transition matrices from the ribbon basis have nonnegative integral coefficients. This is done by means of two composition-valued statistics on permutations and packed words, which generalize the combinatorics of Genocchi numbers.
\end{abstract}

\section{INTRODUCTION}

In the theory of noncommutative symmetric functions [4], the self dual commutative Hopf algebra Sym of ordinary symmetric functions is replaced by a pair of mutually dual Hopf algebras (Sym, QSym), respectively called Noncommutative Symmetric Functions, and Quasi-symmetric functions. The usual bases of Sym are usually lifted only on one side (with the notable exception of Schur functions, which admit natural analogs on both sides). In particular, monomial symmetric functions $m_{\mu}$ split into the quasi-monomial functions $M_{I}$ on the quasi-symmetric side, and their dual basis $h_{\mu}$ is lifted on the noncommutative side, in the form of the homogeneous products $S^{I}$.

In [14], the third author has proposed a construction of noncommutative monomial and forgotten symmetric functions, and conjectured positivity properties of certain transition matrices involving the new bases. The purpose of the present article is to prove these conjectures, by providing combinatorial interpretations.

These interpretations rely on new permutations statistics, which generalize the combinatorics related to the Genocchi numbers.

Notations. We shall depart from the notation of [14] and write $\Psi_{I}$ instead of $M^{I}$, and $L_{I}$ instead of $L^{I}$. Other notations are as in [4. See [3] for background on quasideterminants.

Acknowledgments.- This work has been partially supported by Agence Nationale de la Recherche, grant ANR-06-BLAN-0380. The authors would also like to thank the contributors of the MuPAD project, and especially those of the combinat package, for providing the development environment for this research (see [7] for an introduction to MuPAD-Combinat).

Date: November 20, 2018.

2000 Mathematics Subject Classification. 05E05;15A15; 16W30.

Key words and phrases. Noncommutative symmetric functions, quasideterminants, permutation statistics, Genocchi numbers. 


\section{BACKGROUND}

2.1. Noncommutative symmetric functions. Recall that the algebra Sym of noncommutative symmetric functions is a graded free associative algebra over a sequence $S_{n}$ of indeterminates, with $\operatorname{deg} S_{n}=n$. Among other sequences of generators, the noncommutative power sums of the first kind $\Psi_{n}$ are defined by an oriented analog of Newton's recursion, which may be solved in terms of quasideterminants [4, 3]. The following definition [14] refines formulas (39) and (40) of [4], and defines an analog of the monomial basis which extends the $\Psi_{n}$.

Definition 2.1. The noncommutative monomial symmetric function corresponding to a composition $I=\left(i_{1}, \ldots, i_{r}\right)$ is defined as a quasideterminant of an $r$ by $r$ matrix:

$$
r \Psi_{I} \equiv r \Psi_{\left(i_{1}, \ldots, i_{r}\right)}=(-1)^{r-1}\left|\begin{array}{cccccc}
\Psi_{i_{r}} & 1 & 0 & \ldots & 0 & 0 \\
\Psi_{i_{n-1}+i_{r}} & \Psi_{i_{n-1}} & 2 & \ldots & 0 & 0 \\
\vdots & \vdots & \vdots & \vdots & \vdots & \vdots \\
\Psi_{i_{2}+\ldots+i_{r}} & \ldots & \ldots & \ldots & \Psi_{i_{2}} & n-1 \\
\Psi_{i_{1}+\ldots+i_{r}} & \ldots & \ldots & \ldots & \Psi_{i_{1}+i_{2}} & \Psi_{i_{1}}
\end{array}\right|
$$

where $r$ is the length of $I$. In particular,

$$
\Psi_{(n)}=\Psi_{n}, \text { and } \Psi_{1^{r}}=\Lambda_{r}
$$

where $\Lambda_{r}$ is an elementary symmetric function.

The quasideterminants may be recursively evaluated by means of the following generalized Newton relations:

$$
\begin{aligned}
r \Psi_{i_{1}, \ldots, i_{r}} & =\Psi_{i_{1}} \Psi_{i_{2}, \ldots, i_{r}}-\Psi_{i_{1}+i_{2}} \Psi_{i_{3}, \ldots, i_{r}}+\ldots \\
& +(-1)^{s-1} \Psi_{i_{1}+\cdots+i_{s}} \Psi_{i_{s+1}, \ldots, i_{r}}+\cdots+(-1)^{r} \Psi_{i_{1}+\cdots+i_{r}} .
\end{aligned}
$$

From a noncommutative analog of the quasi-monomial basis $M_{I}$, one can define an analog of Gessel's fundamental basis $F_{I}$ by

$$
L_{I}=\sum_{J \succeq I} \Psi_{J}
$$

Define the coefficients $G_{I J}$ by the expansion

$$
R_{I}=\sum_{J} G_{I J} L_{J}
$$

It has been conjectured in [14] that these numbers are nonnegative integers. Our aim is to prove this fact by means of a combinatorial interpretation.

2.2. Free quasi-symmetric functions. Let us fix an infinite ordered alphabet $A=\left\{a_{1}<\cdots<a_{n}<\ldots\right\}$. The standardized word $\operatorname{Std}(w)$ of a word $w \in A^{*}$ is the permutation obtained by iteratively scanning $w$ from left to right, and labelling $1,2, \ldots$ the occurrences of its smallest letter, then numbering the occurrences of the next one, and so on. 
With a permutation $\sigma$, we associate the polynomial

$$
\mathbf{F}_{\sigma}:=\sum_{\operatorname{Std}(w)=\sigma^{-1}} w
$$

These polynomials span a subalgebra of $\mathbb{K}\langle A\rangle$, called FQSym for Free Quasi-Symmetric functions [2]. Note that the field $\mathbb{K}$ is assumed to be of characteristic zero. Their product rule is given by

$$
\mathbf{F}_{\sigma^{\prime}} \mathbf{F}_{\sigma^{\prime \prime}}=\sum_{\sigma \in \sigma^{\prime} \uplus \sigma^{\prime \prime}} \mathbf{F}_{\sigma}
$$

where the shifted shuffle $\sigma^{\prime} \cup \sigma^{\prime \prime}$ of two packed words is defined as

$$
\sigma^{\prime} \uplus \sigma^{\prime \prime}=\sigma^{\prime} \amalg \sigma^{\prime \prime}[|\sigma|]
$$

the $k$-shift $w[k]$ of a word $w$ being obtained by replacing each letter $w_{i}$ by $w_{i}+k$, and $\amalg$ is the usual shuffle product on words defined recursively by

$$
(a u) Ш(b v)=a \cdot(u Ш(b v))+b \cdot((a u) Ш v),
$$

with $u ш \epsilon=\epsilon \amalg u=u$ if $\epsilon$ is the empty word.

We shall make use of the basis $\mathbf{G}_{\sigma}$ of $\mathbf{F Q S y m}$, dual to $\mathbf{F}_{\sigma}$, defined by $\mathbf{G}_{\sigma}:=\mathbf{F}_{\sigma^{-1}}$.

2.3. Word quasi-symmetric functions. The packed word $u=\operatorname{pack}(w)$ associated with a word $w \in A^{*}$ is obtained by the following process. If $b_{1}<b_{2}<\ldots<b_{r}$ are the letters occuring in $w, u$ is the image of $w$ by the homomorphism $b_{i} \mapsto a_{i}$. A word $u$ is said to be packed if $\operatorname{pack}(u)=u$. We denote by PW the set of packed words. With such a word, we associate the polynomial

$$
\mathbf{M}_{u}:=\sum_{\operatorname{pack}(w)=u} w .
$$

These polynomials span a subalgebra of $\mathbb{K}\langle A\rangle$, called WQSym for Word QuasiSymmetric functions [5, 11] (and called NCQSym in [1]), the invariants of the noncommutative quasi-symmetrizing action. Their product rule is given by

$$
\mathbf{M}_{u^{\prime}} \mathbf{M}_{u^{\prime \prime}}=\sum_{u \in u^{\prime} * W u^{\prime \prime}} \mathbf{M}_{u}
$$

where the convolution $u^{\prime}{ }_{W} u^{\prime \prime}$ of two packed words is defined as

$$
u^{\prime} *_{W} u^{\prime \prime}=\sum_{\substack{v, w ; u=v \cdot w \in \mathrm{PW} \\ \operatorname{pack}(v)=u^{\prime}, \operatorname{pack}(w)=u^{\prime \prime}}} u .
$$

\section{A statistic on permutations generalizing Genocchi numbers}

Genocchi numbers (sequence A001469 of [13]) are known to count a large variety of combinatorial objects, among which numerous sets of permutations. Our statistic derives directly from the most classical of those sets: it is the number of permutations of $\mathfrak{S}_{2 n}$ such that each even integer is followed by a smaller integer and each odd integer is either followed by a greater one, or at the last position of the permutation. 
Let us define the Genocchi descent set (G-descent set for short) of a permutation $\sigma \in \mathfrak{S}_{n}$ as

$$
\operatorname{GDes}(\sigma):=\left\{i \in[2, n] \mid \sigma_{j}=i \Longrightarrow \sigma_{j+1}<\sigma_{j}\right\}
$$

In other words, GDes $(\sigma)$ is the set of values of the descents of $\sigma$, different from the usual set $\operatorname{Des}(\sigma)$ which records the positions of the descents of $\sigma$. Astonishingly enough, this G-statistic behaves very differently from the classical descent statistic. From the G-descent set, we define the Genocchi composition of descents (or G-composition, for short) $\mathrm{GC}(\sigma)$ of a permutation, as the integer composition $I$ of $n$ whose descent set is $\{d-1 \mid d \in \operatorname{GDes}(\sigma)\}$.

The following tables represent the G-composition of all permutations of $\mathfrak{S}_{2}, \mathfrak{S}_{3}$, and $\mathfrak{S}_{4}$.

\begin{tabular}{|c|c|}
\hline 2 & 11 \\
\hline \hline 12 & 21 \\
\hline
\end{tabular}

\begin{tabular}{|c|c|c|c|}
\hline 3 & 21 & 12 & 111 \\
\hline \hline 123 & 132 & 213 & 321 \\
& 231 & & \\
& 312 & & \\
\hline
\end{tabular}

\begin{tabular}{|c|c|c|c|c|c|c|c|}
\hline 4 & 31 & 22 & 211 & 13 & 121 & 112 & 1111 \\
\hline \hline 1234 & 1243 & 1324 & 1432 & 2134 & 2143 & 3214 & 4321 \\
& 1342 & 2314 & 2431 & & 3421 & & \\
& 1423 & 3124 & 3142 & & 4213 & & \\
& 2341 & & 3241 & & & & \\
& 2413 & & 4132 & & & & \\
& 3412 & & 4231 & & & & \\
& 4123 & & 4312 & & & & \\
\hline
\end{tabular}

More combinatorial properties of these numbers, including a hook-length formula will be given in [12].

\section{A Sym QUOTIEnT OF FQSym}

Let $\sim$ be the equivalence relation defined by $\sigma \sim \tau$ iff $\operatorname{GC}(\sigma)=\mathrm{GC}(\tau)$. Let $\mathcal{J}$ be the subspace of FQSym spanned by the differences

$$
\left\{\mathbf{F}_{\sigma}-\mathbf{F}_{\tau} \mid \sigma \sim \tau\right\}
$$

Theorem 4.1. $\mathcal{J}$ is a two-sided ideal of FQSym, and the quotient $\mathbf{T}=\mathbf{F Q S y m} / \mathcal{J}$ is isomorphic to Sym as an algebra.

Moreover, let $T_{I}$ be the image in $\mathbf{T}$ of the $\mathbf{F}_{\sigma}$ such that $\mathrm{GC}(\sigma)=I$. Then

$$
T_{I} T_{J}=\sum_{K} C_{I, J}^{K} T_{K}
$$

where $C_{I, J}^{K}$ is computed as follows. Let $K^{\prime}$ and $K^{\prime \prime}$ be the compositions such that $\left|K^{\prime}\right|=|I|$ and either $K=K^{\prime} \cdot K^{\prime \prime}$, or $K=K^{\prime} \triangleright K^{\prime \prime}$. If $K^{\prime}$ is not coarser than $I$ or 
if $K^{\prime \prime}$ is not finer than $J$, then $C_{I, J}^{K}$ is 0 . Otherwise,

$$
C_{I, J}^{K}=\left(\begin{array}{c}
|I|+l(J)-l(I) \\
l(K)-l(I)
\end{array}\right)
$$

Proof - We have to prove that the set (with multiplicities) of G-compositions of the shifted shuffle of two permutations depends only on the G-compositions of the permutations.

Let $\sigma \in \mathfrak{S}_{m}$ and $\tau \in \mathfrak{S}_{n}$ and let $I$ and $J$ be their respective G-compositions. Let $K=\left(k_{1}, \ldots, k_{r}\right)$ be a composition of $m+n$ and let us compute the number of permutations $\mu$ in $\sigma \mathbb{U} \tau$ such that $\mathrm{GC}(\mu)=K$. We shall need the unique compositions $K^{\prime}$ and $K^{\prime \prime}$ such that $K=K^{\prime} \cdot K^{\prime \prime}$ or $K=K^{\prime} \triangleright K^{\prime \prime}$, with $\left|K^{\prime}\right|=|I|$.

Let us now consider which letters can follow the letters $x$ from 1 to $m$ in $\mu$. We have four cases:

(1a) $x$ is a G-descent of $\mu$ and is not a G-descent of $\sigma$,

(2a) $x$ is a G-descent of $\mu$ and is a G-descent of $\sigma$,

(3a) $x$ is not a G-descent of $\mu$ and is not a G-descent of $\sigma$,

(4a) $x$ is not a G-descent of $\mu$ and is a G-descent of $\sigma$.

The first case implies that $K$ cannot be the G-composition of a word in the shifted shuffle of $\sigma$ and $\mu$. Let us now restrict to compositions $K$ such that $K^{\prime}$ is coarser than $I$. The second case implies that $x$ has to be followed in $\mu$ by a letter coming from $\sigma$. The third one implies nothing about $x$. The fourth one implies that $x$ has to be followed in $\mu$ by a letter coming from $\tau$.

Let $f(\sigma)$ be the number of occurrences of the fourth case. Let $g(\sigma)$ be the number of occurrences of the third and fourth cases, plus one.

Let us now consider which letters can follow the letters $x$ from $m+1$ to $m+n$ in $\mu$. We have again four cases:

(1b) $x$ is a G-descent of $\mu$ and is not a G-descent of $\tau[m]$,

(2b) $x$ is a G-descent of $\mu$ and is a G-descent of $\tau[m]$,

(3b) $x$ is not a G-descent of $\mu$ and is not a G-descent of $\tau[m]$,

(4b) $x$ is not a G-descent of $\mu$ and is a G-descent of $\tau[m]$.

The first case implies that $x$ has to be followed in $\mu$ by a letter coming from $\sigma$. The second case implies nothing about $x$. The third one implies that $x$ has to be followed in $\mu$ by a letter coming from $\tau$. The fourth one implies that $K$ cannot be the G-composition of a word in the shifted shuffle of $\sigma$ and $\mu$. We now restrict to compositions $K$ such that $K^{\prime \prime}$ is finer than $J$.

This preliminary analysis proves that the number of permutations $\mu$ with Gcomposition $K$ is equal to the number of ways of separating the letters of $\tau$ in any number of blocks with given necessary separations (case 1b) and necessary nonseparations (case 3b) and put those blocks in the middle of blocks of letters of $\sigma$, themselves separated into this number of blocks with given necessary separations (case 4a), and necessary non-separations (case 2a). The number of such blocks for each pair of permutations depends only on the lengths of their G-compositions, and a fortiori only on their G-compositions, so that our equivalence relation on permutations indeed induces a quotient algebra of FQSym. 
Let us now determine the structure constants of this algebra. The previous remark can be reformulated as follows. We have two cases depending on whether $K=K^{\prime} \cdot K^{\prime \prime}$ or $K=K^{\prime} \triangleright K^{\prime \prime}$.

In the first case, the number of permutations $\tau$ with G-composition $I$ is

$$
\sum_{k=1}^{\max (n, m)}\left(\begin{array}{c}
m-l(I) \\
k-1-\left(l(I)-l\left(K^{\prime}\right)\right)
\end{array}\right)\left(\begin{array}{c}
n \\
l\left(K^{\prime \prime}\right)+1-k
\end{array}\right) .
$$

This sum of binomial coefficients is easily simplified, and one gets

$$
\left(\begin{array}{c}
m-l(I)+l(J) \\
l\left(K^{\prime}\right)+l\left(K^{\prime \prime}\right)-l(I)
\end{array}\right) .
$$

In the second case, the number of permutations $\tau$ with G-composition $I$ is

$$
\sum_{k=1}^{\max (n, m)}\left(\begin{array}{c}
m-l(I) \\
k-1-\left(l(I)-l\left(K^{\prime}\right)\right.
\end{array}\right)\left(\begin{array}{c}
n \\
l\left(K^{\prime \prime}\right)-k
\end{array}\right) .
$$

Similarly, this sum of binomial coefficients reduces to

$$
\left(\begin{array}{c}
m-l(I)+l(J) \\
l\left(K^{\prime}\right)+l\left(K^{\prime \prime}\right)-1-l(I)
\end{array}\right)
$$

so that $C_{I J}^{K}$ is indeed given by (18) in any case.

One can notice that these coefficients coincide, in the special case $I=(n)$ with those of the product $L_{I} L_{J}$ (see Proposition 4.6 of [14]), so that, since the $L_{n}$ are algebraic generators of Sym, the $T_{n}$ are algebraic generators of $\mathbf{T}$. Moreover, since Sym is free over the sequence $L_{n}=S_{n}$, the algebra $\mathbf{T}$ is free over the $T_{n}$.

Example 4.2. Let $I=(2,2,1), J=(1,3)$, and $K=(4,2,1,1,1)$. We can choose $\sigma=32514$ and $\tau=2134$.

We then have $K^{\prime}=(4,1)$ and $K^{\prime \prime}=(1,1,1,1)$. The coefficient of $K$ in $T_{I} T_{J}$ is $\left(\begin{array}{c}5+2-3 \\ 5-3\end{array}\right)=6$ and, indeed, there are six permutations in the shifted shuffle $32514 \cup 2134$ with G-composition $K$ :

$$
\text { 372685194, 376825194, 376829514, 736825194, 736829514, 768392514. }
$$

Those six permutations are obtained as follows: $\sigma$ has one necessary separation between 3 and 2 and one necessary non-separation between 1 and 5 , and nothing after 4 . The permutation $\tau[5]$ has two necessary separations, between 8 and 9 , and after 9 , and one necessary non-separation between 6 and 8 . Then one inserts the blocks of $\tau[5]$ in $\sigma$, satisfying the separation/non-separation constraints and gets the six permutations.

Note 4.3. Note that this quotient is not a Hopf quotient, since as one can easily check, $\mathcal{J}$ is not a coideal. For example, $231 \sim 312$ but

$$
\bar{\Delta}\left(\mathbf{F}_{231}\right)=\mathbf{F}_{12} \otimes \mathbf{F}_{1}+\mathbf{F}_{1} \otimes \mathbf{F}_{21} \text {, and } \bar{\Delta}\left(\mathbf{F}_{312}\right)=\mathbf{F}_{21} \otimes \mathbf{F}_{1}+\mathbf{F}_{1} \otimes \mathbf{F}_{12} \text {. }
$$




\section{Change of Bases in Sym}

Thanks to the previous result, we have a map going from Sym to itself in a very unusual way: start with the injection of Sym into FQSym*, and compose it with the self duality isomorphism of FQSym, which reads

$$
R_{I}:=\sum_{\mathrm{D}(\sigma)=I} \mathbf{G}_{\sigma}=\sum_{\mathrm{D}\left(\tau^{-1}\right)=I} \mathbf{F}_{\tau},
$$

where $\mathrm{D}$ is the composition whose descent set is equal to the descent set of $\sigma$, and then go from FQSym to Sym by the G-quotient homomorphism.

Let $\phi$ be the composition of those maps and let $R_{I}^{\prime}$ be the image of $R_{I}$ by $\phi$ :

$$
\begin{aligned}
\phi: \text { Sym } & \rightarrow \mathbf{T} \\
R_{I} & \mapsto R_{I}^{\prime} .
\end{aligned}
$$

By definition of $\phi$, we have

$$
R_{I}^{\prime}:=\sum_{\mathrm{D}\left(\sigma^{-1}\right)=I} \overline{\mathbf{F}_{\sigma}}=\sum_{\substack{\mathrm{D}(\sigma-1)=I \\ \mathrm{GC}(\sigma)=J}} T_{J}
$$

where $\overline{\mathbf{F}}_{\sigma}$ is the image of $\mathbf{F}_{\sigma}$ by the G-quotient homomorphism. Then, since $L_{n}=R_{n}$ and $R_{n}^{\prime}=\overline{\mathbf{F}_{12 \ldots n}}=T_{n}$, we have $\phi\left(L_{n}\right)=T_{n}$ for all $n$, so that, thanks to the product formulas of $L_{n}$ and $T_{n}, \phi\left(L_{I}\right)=T_{I}$ for all compositions $I$.

Since the $T_{n}$ are algebraic generators of $\mathbf{T}$, the algebra morphism $\phi$ is an isomorphism of algebras, so that, applying $\phi^{-1}$ to Equation (27), one gets

Theorem 5.1. Let $I$ be a composition of $n$. Then

$$
R_{I}=\sum_{J \models n} G_{I J} L_{J}
$$

where $G_{I J}$ is the number of permutations satisfying $\mathrm{D}\left(\sigma^{-1}\right)=I$ and $\mathrm{GC}(\sigma)=J$. In particular, the $G_{I J}$ are nonnegative integers.

Examples of the transition matrices are given in Section 7.1, together with the same matrices filled with the corresponding permutations. The Genocchi numbers appear as the sums of the values in the rows indexed by compositions of the form $\left(2^{n}\right)$ or $\left(2^{n} 1\right)$.

Combining this last result with Equation (44), one then gets

Corollary 5.2. Let $I$ be a composition of $n$. Then

$$
R_{I}=\sum_{J \models n} K_{I J} \Psi_{J}
$$

where $K_{I J}$ are nonnegative integers.

One can easily describe those integers in terms of permutations. They can be described in a much more natural way in terms of packed words as one shall see in the following section. 
Note 5.3. Theorems 17 and 5.1 prove Conjecture 4.1 of [14]. Theorem 5.1 proves Conjecture 5.4 of [14]. Corollary 5.2 proves Conjecture 5.3 of [14]. Corresponding statements for the "forgotten" basis are obtained by applying the canonical involution $\omega$.

\section{Permutations Replaced By PaCKed WORds}

The previous section was devoted to the study of the transition matrices from $R$ to $L$, we now apply a similar analysis to the transition matrices from $R$ to $\Psi$. As already mentioned, the latter can be described in terms of the former, since there is a very simple transition matrix from $L$ to $\Psi$ : it is the matrix of the refinement order on compositions. Nevertheless, as the sum of the entries of the transition matrix $M_{n}(R, L)$ is $n$ !, the sum of the entries of a transition matrix $M_{n}(R, \Psi)$ is the $n$th ordered Bell number (sequence A000670 of [13]) counting, for example, set compositions (ordered set partitions), or packed words.

This suggests the existence of two statistics on packed words giving back the entries of the transition matrices, exactly as in the $(R, L)$ case. The algebraic context is essentially the same as before if one replaces the algebra FQSym by the algebra WQSym (see [5, 11]).

The proof of the connection between the two statistics and the matrices $M(R, \Psi)$ follows the same guidelines as the previous proof. We first define a compositionvalued statistic on packed words, then prove that this statistic defines a quotient of WQSym, isomorphic to Sym as an algebra. Then, comparing the structure constants of the natural base with those of the $\Psi_{I}$, we prove that they are mapped to each other by a simple isomorphism, hence giving the coefficients of the matrix $M(R, \Psi)$.

6.1. A statistic on packed words. Let $w$ be a packed word. The Word composition (W-composition) of $w$ is the composition whose descent set is given by the positions of the last occurrences of each letter in $w$. For example,

$$
\mathrm{WC}(1543421323)=(2,3,2,2,1) .
$$

Indeed, the descent set is $\{2,5,7,9,10\}$ since the last 5 is in position 2 , the last 4 is in position 5 , the last 1 is in position 7 , the last 3 is in position 9 , the last 2 is in position 10 .

The following tables represent the $\mathrm{W}$-compositions of all packed words in $\mathrm{PW}_{2}$ and $\mathrm{PW}_{3}$. One can recover from the matrix $\mathfrak{M}_{4}^{\prime}$ in Section 7.2 the $\mathrm{W}$-compositions in $\mathrm{PW}_{4}$ : it is the composition indexing their row.

\begin{tabular}{|c|c|}
\hline 2 & 11 \\
\hline \hline 11 & 12 \\
& 21 \\
\hline
\end{tabular}

\begin{tabular}{|c|c|c|c|}
\hline 3 & 21 & 12 & 111 \\
\hline \hline 111 & 112 & 122 & 123 \\
& 121 & 211 & 132 \\
& 212 & & 213 \\
& 221 & & 231 \\
& & & 312 \\
& & & 321 \\
\hline
\end{tabular}


6.2. A Sym quotient of WQSym. Let $\sim$ be the equivalence relation on packed words defined by $u \sim v$ iff $\mathrm{WC}(u)=\mathrm{WC}(v)$. Let $\mathcal{J}^{\prime}$ be the subspace of WQSym spanned by the differences

$$
\left\{\mathbf{M}_{u}-\mathbf{M}_{v} \mid u \sim v\right\} .
$$

Theorem 6.1. $\mathcal{J}^{\prime}$ is a two-sided ideal of WQSym, and the quotient $\mathbf{T}^{\prime}$ defined by $\mathbf{T}^{\prime}=\mathbf{W Q S y m} / \mathcal{J}^{\prime}$ is isomorphic to $\mathbf{S y m}$ as an algebra.

Moreover, let $U_{I}$ be the image of $\mathbf{M}_{u}$ in $\mathbf{T}^{\prime}$. Then

$$
U_{I} U_{J}:=\sum_{K} D_{I, J}^{K} U_{K}
$$

where $D_{I, J}^{K}$ is computed as follows. Let $K^{\prime}$ and $K^{\prime \prime}$ be the compositions such that $\left|K^{\prime}\right|=|I|$ and either $K=K^{\prime} \cdot K^{\prime \prime}$, or $K=K^{\prime} \triangleright K^{\prime \prime}$. If $K^{\prime}$ is not coarser than $I$, then $D_{I, J}^{K}$ is 0 . Otherwise,

$$
D_{I, J}^{K}=\left(\begin{array}{c}
l(K) \\
l(I)
\end{array}\right)
$$

Proof - The proof follows essentially the same lines as the proof of Theorem4.1, so we only sketch it. In fact, the details are much simpler than for Theorem 4.1, Looking at the definitions of the $\mathrm{W}$-composition and of the convolution of packed words, it is clear that the multiset of the W-compositions of the words in the convolution of two packed words depends only on the W-compositions of the words. So the product is well-defined and $\mathbf{T}^{\prime}$ is a quotient of WQSym.

Let us now see why the product $U_{I} U_{J}$ is given by Equations (33) and (34). Let us choose two words $u$ and $v$ such that $\mathrm{WC}(u)=I$, and $\mathrm{WC}(v)=J$. Since there is exactly one nondecreasing word having a given $\mathrm{WC}$, we can assume that $u$ and $v$ are nondecreasing. Let $|v|$ be the size of $v$. Let us compute $\mathbf{M}_{u} \mathbf{M}_{v}$.

The idea is that a word $w \in u *_{W} v$ satisfies $\mathrm{WC}(w)=K$ iff the last $|v|$ letters of $w$ have specific values, depending on $K^{\prime}$. Indeed, by definition of $\mathrm{WC}$, if $K^{\prime}$ is not coarser than $I$, or if $K^{\prime \prime} \neq J$, then the coefficient of $U_{K}$ is zero. Now, let us fix a composition $I=\left(i_{1}, \ldots, i_{l}\right)$ and a composition $K$ satisfying the conditions of the theorem. Then any word of $u *_{W} v=w=u^{\prime} \cdot v^{\prime}$ satisfying $\operatorname{WC}(w)=K$ has also $|K|$ different letters. Now, for all $j<l$, if $I_{j}$ and $I_{j+1}$ come from the same part of $K$, the letter $u_{i_{1}+\cdots+i_{j}}^{\prime}$ has to appear in $v^{\prime}$, otherwise this letter does not appear in $v^{\prime}$. Hence, given the letters appearing in $u^{\prime}$, the letters appearing in $v^{\prime}$ are also fixed, which completely determines $v^{\prime}$ too (since its packed word is given). The number of ways of choosing the letters appearing in $u^{\prime}$ obviously is the binomial coefficient $\left(\begin{array}{c}l(K) \\ l(I)\end{array}\right)$.

Example 6.2. Let $I=(2,2,1), J=(1,3)$, and $K=(4,1,1,3)$. We can choose $\sigma=23212$ and $\tau=2111$. Then $K^{\prime}=(4,1)$ and $K^{\prime \prime}=(1,3)$. The coefficient of $K$ in $U_{I} U_{J}$ is $\left(\begin{array}{l}4 \\ 2\end{array}\right)=6$ and, indeed, there are four packed words in the (modified) convolution $11223 *_{W} 1222$ with W-composition $K$ :

112241333, 113341222, 112231444, 223341222. 
Those four packed words are obtained as follows: since $(4,1,1,3)$ is obtained by gluing together the first two parts of $I$, this means that, if $\mathrm{WC}(w)=K$, the last four letters of $w$ have to be the first letter of $w$ or the one not used in its first five letters.

Note 6.3. This quotient is not a Hopf quotient, since again, $\mathcal{J}^{\prime}$ is not a coideal. For example, $221 \sim 112$ but

$$
\bar{\Delta}\left(\mathbf{M}_{221}\right)=\mathbf{M}_{1} \otimes \mathbf{M}_{11} \text {, and } \bar{\Delta}\left(\mathbf{M}_{112}\right)=\mathbf{M}_{11} \otimes \mathbf{M}_{1} .
$$

6.3. Change of bases in Sym. As in the case of permutations, we have a map going from Sym to itself: start with the injection of Sym into WQSym, which reads

$$
R_{I}:=\sum_{\mathrm{D}(u)=I} \mathbf{M}_{u}
$$

and then go from WQSym to Sym by the W-quotient homomorphism.

Let $\phi^{\prime}$ be the composition of those maps and let $R_{I}^{\prime}$ be the image of $R_{I}$ by $\phi^{\prime}$ :

$$
\begin{aligned}
\phi^{\prime}: \text { Sym } & \rightarrow \mathrm{T}^{\prime} \\
R_{I} & \mapsto R_{I}^{\prime} .
\end{aligned}
$$

By definition of $\phi^{\prime}$, we have

$$
R_{I}^{\prime}:=\sum_{\mathrm{D}(u)=I} \overline{\mathbf{M}_{u}}=\sum_{\substack{\mathrm{D}(u)=I \\ \mathrm{WC}(u)=J}} \Psi_{J},
$$

where $\overline{\mathbf{M}}_{u}$ is the image of $\mathbf{M}_{u}$ by the $\mathrm{W}$-quotient homomorphism. Then, since $\Psi_{1^{n}}=$ $R_{1^{n}}$ and $R_{1^{n}}^{\prime}=\overline{\mathbf{M}_{n \ldots 21}}=U_{1^{n}}$, we have $\phi^{\prime}\left(\Psi_{n}\right)=U_{n}$ for all $n$, so that, thanks to the product formulas of $\Psi_{n}$ and $U_{n}, \phi^{\prime}\left(\Psi_{I}\right)=U_{I}$ for all compositions $I$.

Since the $U_{n}$ are algebraic generators of $\mathbf{T}^{\prime}$, the algebra morphism $\phi^{\prime}$ is an isomorphism of algebras, so that, applying $\phi^{\prime-1}$ to Equation (39), one gets

Theorem 6.4. Let I be a composition of $n$. Then

$$
R_{I}=\sum_{J \models n} K_{I J} \Psi_{J}
$$

where $K_{I J}$ is the number of permutations satisfying $\mathrm{D}(u)=I$ and $\mathrm{WC}(u)=J$. In particular, the $K_{I J}$ are nonnegative integers.

Combining this last result with Equation (4), one then gets back Corollary 5.2. Examples of the transition matrices are given in Section [7.2, together with the same matrices filled with the corresponding packed words.

\section{TABLES}

7.1. Coefficients $G_{I J}$. Here are the transition matrices from $R$ to $L$ (the matrices of the coefficients $G_{I J}$ ) for $n=3$ and $n=4$, the compositions being in lexicographic order. To save space and for better readability, 0 has been represented by a dot. 


$$
\begin{aligned}
& M_{3}(R, L)=\left(\begin{array}{cccc}
1 & \cdot & \cdot & \cdot \\
\cdot & 2 & 1 & \cdot \\
\cdot & \cdot & 1 & \cdot \\
\cdot & \cdot & \cdot & 1
\end{array}\right) \\
& M_{4}(R, L)=\left(\begin{array}{cccccccc}
1 & . & . & . & . & . & . & . \\
. & 3 & 2 & . & 1 & 1 & . & . \\
. & . & 2 & . & 1 & . & . & . \\
. & . & 1 & 3 & . & 2 & 1 & . \\
. & . & . & . & 1 & . & . & . \\
. & . & . & . & . & 2 & 1 & . \\
. & . & . & . & . & . & 1 & . \\
. & . & . & . & . & . & . & 1
\end{array}\right)
\end{aligned}
$$

Here are the same matrices with the list of permutations having a given recoil composition (or descent composition of the inverse) and G-composition, instead of their number.

$\mathfrak{M}_{3}=$\begin{tabular}{|c||c|c|c|c|}
\hline GC $\backslash$ Rec & 3 & 21 & 12 & 111 \\
\hline \hline 3 & 123 & & & \\
\hline 21 & & 132 & 231 & \\
\hline 12 & & & 213 & \\
\hline 111 & & & & 321 \\
\hline
\end{tabular}

\begin{tabular}{|c||c|c|c|c|c|c|c|c|}
\hline GC $\backslash$ Rec & 4 & 31 & 22 & 211 & 13 & 121 & 112 & 1111 \\
\hline \hline 4 & 1234 & & & & & & & \\
\hline 31 & & $\begin{array}{c}1243,1423 \\
4123\end{array}$ & $\begin{array}{c}1342 \\
3412\end{array}$ & & 2341 & 2413 & & \\
\hline 22 & & & $\begin{array}{c}1324 \\
3124\end{array}$ & & 2314 & & & \\
\hline 211 & & & 3142 & $\begin{array}{c}1432,4132 \\
4312\end{array}$ & & $\begin{array}{c}2431 \\
4231\end{array}$ & 3241 & \\
\hline 13 & & & & & 2134 & & & \\
\hline 121 & & & & & & 2143 & 3421 & \\
\hline 112 & & & & & & & 3214 & \\
\hline 1111 & & & & & & & & 4321 \\
\hline
\end{tabular}

7.2. Coefficients $K_{I J}$. Here are the transition matrices from $R$ to $\Psi$ (the matrices of the coefficients $K_{I J}$ ) for $n=3$ and $n=4$, the compositions being in lexicographic order. To save space and for better readability, 0 has been represented by a dot. 


$$
M_{3}(R, \Psi)=\left(\begin{array}{cccc}
1 & . & . & . \\
1 & 2 & 1 & . \\
1 & . & 1 & . \\
1 & 2 & 2 & 1
\end{array}\right)
$$

Here are the same matrices with the list of packed words having a given descent composition and W-composition, instead of their number.

$\mathfrak{M}_{3}^{\prime}=$\begin{tabular}{|c||c|c|c|c|}
\hline WC $\backslash \mathrm{D}$ & 3 & 21 & 12 & 111 \\
\hline \hline 3 & 111 & & & \\
\hline 21 & 112 & 121 & 212 & \\
\hline 12 & 122 & & 211 & \\
\hline 111 & 123 & 132 & 312 & 321 \\
\hline
\end{tabular}

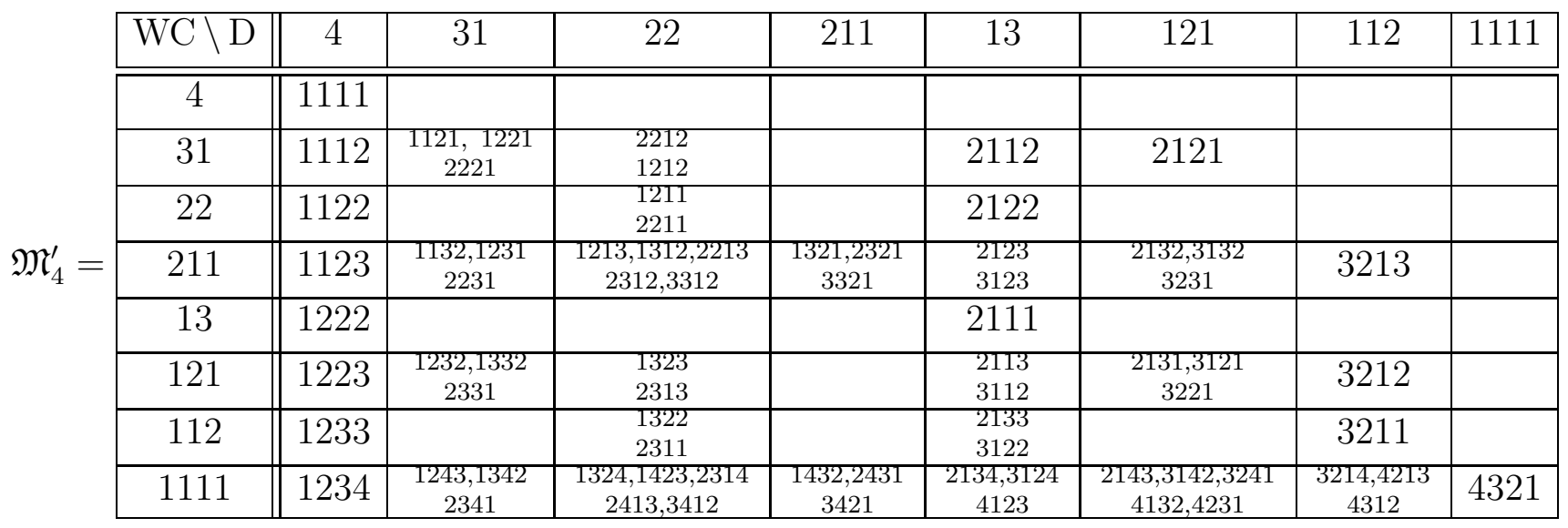

REFERENCES

[1] N. Bergeron and M. ZABRoCKI, The Hopf algebras of symmetric functions and quasisymmetric functions in non-commutative variables are free and cofree, preprint math.CO/0509265.

[2] G. Duchamp, F. Hivert, and J.-Y. Thibon, Noncommutative symmetric functions VI: free quasi-symmetric functions and related algebras, Internat. J. Alg. Comput. 12 (2002), 671-717.

[3] I. Gelfand, S. Gelfand, V. Retakh, and R. L. Wilson, Quasideterminants, Adv. Math. 193 (2005), 56-141.

[4] I.M. Gelfand, D. Krob, A. Lascoux, B. Leclerc, V. S. Retakh, and J.-Y. Thibon, Noncommutative symmetric functions, Adv. in Math. 112 (1995), 218-348. 
[5] F. Hivert, Combinatoire des fonctions quasi-symétriques, Thèse de Doctorat, Marne-LaVallée, 1999.

[6] F. HiverT, Hecke Algebras, Difference Operators, and Quasi-Symmetric Functions, Advances in Math. 155 (2000), 181-238.

[7] F. Hivert and N. ThiÉRY, MuPAD-Combinat, an open-source package for research in algebraic combinatorics, Sém. Lothar. Combin. 51 (2004), 70p. (electronic).

[8] D. Krob, B. LeClerC and J.-Y. ThiBon, Noncommutative symmetric functions II: Transformations of alphabets, Intern. J. Alg. Comput. 7 (1997), 181-264.

[9] D. KroB and J.-Y. ThiBon, Noncommutative symmetric functions IV: Quantum linear groups and Hecke algebras at $q=0$, J. Alg. Comb. 6 (1997), 339-376.

[10] I.G. Macdonald, Symmetric functions and Hall polynomials, 2nd ed., Oxford University Press, 1995.

[11] J.-C. Novelli and J.-Y. Thibon, Polynomial realizations of some trialgebras, Proc. FPSAC'06, San Diego, USA.

[12] J.-C. Novelli, J.-Y. Thibon, and L. K. Williams, to be written.

[13] N.J.A. SloAne, The On-Line Encyclopedia of Integer Sequences, http://www.research.att.com/ ${ }^{\sim}$ njas/sequences/

[14] L. Tevlin, Noncommutative Monomial Symmetric Functions, Proc. FPSAC'07, Tianjin, China.

(F. Hivert) LiTIS, Université de Rouen ; Avenue De L'Université ; 76801 Saint Étienne Du Rouvray, France,

(J.-C Novelli, J.-Y. Thibon) Institut Gaspard Monge, Université Paris-Est Marne-LaVallée, 5 Boulevard Descartes, Champs-sur-Marne, 77454 Marne-la-Vallée cedex 2, FRANCE

(L. Tevlin) Physics Department, Yeshiva University, 500 West 185th Street, New YoRK, N.Y. 10033, USA

E-mail address, F. Hivert: hivert@univ-rouen.fr

E-mail address, J.-C. Novelli: novelli@univ-mlv.fr

E-mail address, L. Tevlin: tevlin@yu.edu

E-mail address, J.-Y. Thibon: jyt@univ-mlv.fr 